\section{Analysis of 372 Patients with Crush Syndrome Jun Oda}

Department of Acute Critical Medicine, Osaka University Medical School, Osaka, Japan

To clarify clinical features and determine the severity in patients with crush syndrome from the Hanshin-Awaji earthquake, medical records of 6,107 patients hospitalized in 95 hospitals were reviewed retrospectively, and 372 patients with crush syndrome were identified. The major sites of crush injury were in the lower extremities (74\%), the upper extremities (10\%), and the trunk (9\%). Pelvic fractures, limb fractures, and abdominal injuries were the most frequently associated injuries. Patients with trunk compression and/or with abdominal injury had a higher mortality rate. A total of 50 patients (13.4\%) died. The causes of death within five days after the earthquake were hypovolemia and hyperkalemia. Peak serum creatine kinase (CK) concentration increased with the number of crushed extremities. Mortality and the risk of acute renal failure were highest in patients with $\mathrm{CK}$ concentration greater than $75,000 \mathrm{U} / \mathrm{L}$.

Conclusion: The severity of crush syndrome can be estimated by both the number of crushed limbs and peak serum CK concentration, which reflects the extent of underlying muscle damage. These results help us to recognize the crush syndrome, evaluate its severity, and to consider indications for transferring the patients to unaffected hospitals for critical management.

Keywords: creatine kinase (CK); crush syndrome; hyperkalemia; hypovolemia; mortality; risk; severity; sites

Prehosp Disast Med 2002;17:s25.

\section{Diagnosis, Treatment, and Pathophysiology of the Crush Syndrome \\ Junichiro Yokota}

Senshu Critical Care Medical Center, Japan

Crush syndrome with acute renal failure has been identified as a major medical complication that occurs among people who are trapped under the debris from earthquakes, bombings, and other events that result in entrapment. Traumatic rhabdomyolysis induced by ischemia reperfusion injury plays a crucial role, although the pathophysiology is not fully understood. Initially, prolonged limb compression may cause stretch myopathy as well as ischemic injury. Immediately following decompression of limbs, re-establishment of the microcirculation produces an adverse effect; reperfusion injury that promotes further ischemia, muscle swelling, and myonecrosis that leads to an acute compartment syndrome. In association with these local events during limb compression/decompression, a massive loss of extracellular fluid into the injured muscles and solutes leaking out of damaged muscles results in the development of systemic manifestation. Chief among these are hypovolemia and hyperkalemia which, synergistically, have a potential for early death, metabolic acidosis, shock, coagulopathy, and acute renal failure (ARF).
A history of prolonged limb compression trapped by heavy objects and a physical finding of limb paralysis following extrication should suggest the diagnosis of crush syndrome. The treatment consists of aggressive volume replacement followed by forced diuretic therapy, which may combat shock and correct the hyperkalemia. If ARF has occurred, regular hemodialysis is indicated. Although the surgical management of injured limbs still is controversial, suggestions were provided in this report.

Keywords: acidosis; acute renal failure; crush syndrome; hemodialysis; ischemia; myonecrosis; reperfusion; thabdomyolysis; shock

Prehosp Disast Med 2002;17:s25.

\section{Radiographic Imaging and Histological Findings of Crush Syndrome \\ Masashi Kishi, Tetsuro Nishimura \\ Department of Emergency and Critical Care Medicine, Osaka Police Hospital, Osaka, Japan}

Objective: To identify the radiographic imaging and histological findings of crush syndrome to better understand the pathophysiology of its development.

Radiographic findings: Seven patients injured in the Hanshin-Awa Earthquake underwent serial computerized tomography $(\mathrm{CT})$ and magnetic resonance imaging (MRI) examinations of the injured extremities. In all patients, the CT showed persistent edema of the subcutaneous tissue until approximately one month after the injury. In four patients who developed acute renal failure, CT revealed transient high density areas in the muscle, which was confirmed histologically as calcification. An MRI performed 4 to 5 weeks after the injury revealed inhomogeneous high intensity areas in injured muscles on T2-weighted images in all patients, indicating prolonged edema within the muscles. The use of Contrast T1-weighted MRI localized the injured area more clearly than did CT.

Histological findings: Muscle tissue was taken by biopsy from six patients 40 to 50 days after their injury. In four patients, typical myopathic changes such as necrosis, regeneration, and inflammatory cell infiltration were observed. Neurogenic muscular changes, such as small angular fibrosis, also were observed in three patients. In five patients, calcium deposition was demonstrated by von Kossa staining.

Conclusions: These radiological and histological findings support the notion of calcium-related biochemical events intrinsic to crush syndrome and show that the mechanism of the injury is not simple, but sustained and complicated. Keywords: calcifications; computerized tomography; edema; fibrosis; histology; imaging; inflammation; magnetic resonance imaging; myonecrosis; necrosis

Prehosp Disast Med 2002;17:s25.

\section{Clinical Presentations of Typical and Atypical Crush Syndrome \\ Yasuki Nakata; ${ }^{1}$ Mitsuo Obnish; ${ }^{1}$ Takeshi Shimazu; ${ }^{2}$ \\ Toshibaru Yoshioka, ${ }^{3}$ Hisashi Sugimoto ${ }^{2}$ \\ 1. Traumatology and Critical Care Medical Center, Osaka National Hospital, Osaka, Japan}


2. Department of Traumatology and Acute Critical Medicine, Osaka, University Medical School, Osaka, Japan

3. Department of Emergency Medicine, Osaka Prefectural General Hospital, Osaka, Japan

A total of 14 patients with crush syndrome were transferred to Osaka University Hospital after the 1995 Hanshin-Awaji earthquake. Clinical aspects of three cases representing the typical and atypical crush syndrome were presented.

[Case 1] A 66-year-old female was buried under a collapsed house and was rescued one hour after entrapment. She was conscious on the scene and was evaluated as yellow (severe, but not critical). However, she developed cardiopulmonary arrest during transport to the hospital. She was successfully resuscitated, and was referred to this Center. She had unstable pelvic fractures as well as crush injury to the buttock. Bleeding from the pelvic fracture was the cause of her sudden collapse. Transcatheter embolization controlled the bleeding and external fixation of the pelvis was provided. She did not develop renal failure or other organ dysfunctions. In this patient, the crush injury was not severe, but the associated pelvic fracture and hemorrhage caused critical situation.

[Case 2] A 25-year-old female was buried under the debris for three hours and was transferred to this hospital three hours following her extrication. On admission, severe hyperkalemia, severe rhabdomyolysis, and increased intracompartmental pressure were detected. Emergency hemodialysis and fasciotomy were performed. Aggressive fluid supplementation reaching 24 liters for the initial 24 hours was needed to counteract massive edema and fluid loss to the injured legs. This fluid support was sustained until the fasciotomy wound was closed. The clinical course was not complicated by acute renal failure, and now she can walk without assistance.

[Case 3] A 25-year-old male was transferred to this hospital 35 hours after injury. He had been compressed under the debris for eight hours, and had renal failure on admission. He was hemodialyzed from the day of arrival. After several days, he had unexpected complications including necrosis of the gall bladder that required surgical intervention. His general condition and renal function recovered rapidly after the operation.

Keywords: acute renal failure; cardiopulmonary arrest; crush syndrome; earthquake; fasciotomy; fracture; hemodialysis; hyperkalemia; rhabdomyolysis

Prehosp Disast Med 2002;17:s25-26.

\section{Long-Term Physical Outcome of Patients Who Suffered Crush Syndrome: Prognostic Indicators Tetsuya Matsuoka \\ Senshu Critical Care Medical Center, Japan}

Objective: To identify independent predictors of physical outcome in patients suffering from crush syndrome Methods: Sensory and motor functions were examined two years after the 1995 Hanshin-Awaji earthquake in 42 patients with a combined total of 58 compressed lower extremities. The influences of time to rescue, fasciotomy, and radical debridement on lower leg muscle strength were evaluated by stepwise regression analysis.

Results: Severe disabilities related to the lower leg compartment were present, and the anterior compartment was damaged more severely than was the posterior compartment. Stepwise regression analysis showed the performance of fasciotomy and debridement to be an independent predictor of long-term, lower leg muscle survival, and indicated that it was an independent predictor when the debrided compartments were not included in the analysis. In all debrided anterior compartments, muscle contractility was abolished completely. There was a significant negative correlation between time to fasciotomy and lower leg muscle strength. Conclusions: Secondary compartment syndrome affected physical outcome in crush syndrome patients. There was no evidence that fasciotomy improves functional outcome. Delayed rescue, delayed fasciotomy, and radical debridement may worsen the physical prognosis. Indications for fasciotomy in crush syndrome during the acute phase need further study and evaluation.

Keywords: debridement; earthquake; fasciotomy; Hanshin-Awaji; morbidity; predictors; rescue

Prehosp Disast Med 2002;17:s26.

\section{Penetrating Cardiac Injury following Sternal Fracture}

Francis Fu Sbeng $W_{u} ;^{1}$ Chi-Wen Juan; ${ }^{1}$ Fang-Cbu Cben; ${ }^{1}$ Sai-Kit Ho;1, Yao-Ru Hu; ${ }^{2}$ Tzu-Hui Tung ${ }^{2}$

1. Department of Emergency Medicine, Changhua Christian Hospital, Changua, Taiwan, ROC

2. Department of Nursing, Changhua Christian Hospital, Changua, Taiwan, ROC

Objective: Myocardial injury used to be considered as one of the major complications associated with sternal fracture, even though recent studies on injuries associated with fracture of sternum are contrary to this belief. Many authors now believe the presence of sternal fracture no longer is indicative of occult injuries to the underlying structure such as the heart.

Methods: A case was presented of a 38-year-old female patient transferred to our hospital after being injured in a motor vehicle accident. On arrival, her blood pressure (BP) was $90 / 50 \mathrm{mmHg}$ but two hours later, it dropped to $60 / 30$ $\mathrm{mmHg}$. Although her chest roentgenography and electrocardiography (ECG) did not reveal any significant findings, chest computerized tomography (CT) scan later revealed a sternal fracture and cardiac tamponade.

Results: A diagnosis of cardiac rupture resulting from sternal fracture following blunt chest trauma was made. Under midline sternotomy, her right atrial rupture was repaired. The patient was doing well during a three months postoperative follow-up.

Conclusion: Clinicians should maintain a high index of suspicion for the presence cardiac tamponade in cases presented as blunt chest trauma as early diagnosis and surgical intervention is vital to the patient's survival. 